4. Liljedahl, M. et al. Cell 104, 409-420 (2001).

5. Yeaman, C. et al. Nature Cell Biol. 6, 106-112 (2004).

6. Hausser, A. et al. Nature Cell Biol. 7, 880-886 (2005).

7. Hama, H. et al. J. Biol. Chem. 274, 34294-34300 (1999).

8. Walch-Solimena, C. \& Novick, P. Nature Cell Biol. 1,
523-525 (1999).

9. Audhya, A. et al. Mol. Biol. Cell 11, 2673-2689 (2000).

10. Godi, A. et al. Nature Cell Biol. 1, 280-287 (1999).

11. Bruns, J.R., Ellis, M.A., Jeromin, A. \& Weisz, O.A. J. Biol. Chem. 277, 2012-2018 (2002).

12. Doppler, H. et al. J. Biol. Chem. 280, 1501315019 (2005).
13. Godi, A. et al. Nature Cell Biol. 6, 393-404 (2004)

14. de Graaf, P. et al. Mol. Biol. Cell. 15, 2038-2047 (2004).

15. Wang, Y. J. et al. Cell 114, 299-310 (2003).

16. Bonazzi, M. et al. Nature Cell Biol. 7, 570-580 (2005).

\section{Mitochondrial wrinkles: the first signs of ageing?}

Although the process of ageing is thought to be conserved, we are only starting to understand some of the molecular mechanisms underlying it. DNA damage is likely to be an important contributor to ageing, and mutations in mitochondrial DNA (mtDNA) have been shown to accumulate with age in several species. Kujoth et al. now report in Science $(309,481-484 ; 2005)$ on some of the cellular mechanisms by which mtDNA damage contributes to ageing.

The authors engineered knock-in mice expressing a mutant of the mtDNA polymerase POLG, which is impaired for DNA proofreading. These mice exhibit a premature ageing phenotype beginning at 9 months, which includes age-related hearing loss and reduction in skeletal muscle. POLG-defective mice had accumulated mtDNA mutations at a frequency of three to eight times that of wild-type littermates.

According to the free radical theory, ageing is due to the accumulation of reactive oxygen species (ROS), which inflict tissue damage including mtDNA mutations. This results in a self-sustained feed-forward loop, because one of the important functions of mitochondria is to clear ROS from cells. To test whether ROS accumulation contributes to the ageing phenotype, Kujoth and colleagues measured the levels of $\mathrm{H}_{2} \mathrm{O}_{2}$ and protein carbonyls in mitochondria of the heart and liver, but did not observe a difference between POLG-defective and wild-type mice. Levels of lipid peroxidation or RNA and DNA oxidative damage were also similar between mutant and wild-type mice, suggesting that oxidative stress does not contribute to the ageing phenotype seen in POLG-defective mice. In addition, the authors were able to exclude an intrinsic defect in cell proliferation or senescence as the likely cause of accelerated ageing.

During apoptosis, mitochondrial dysfunction leads to permeabilization of their outer membrane and the release of cytochrome $c$ in the cytosol. This activates caspase- 3 and amplifies the programmed

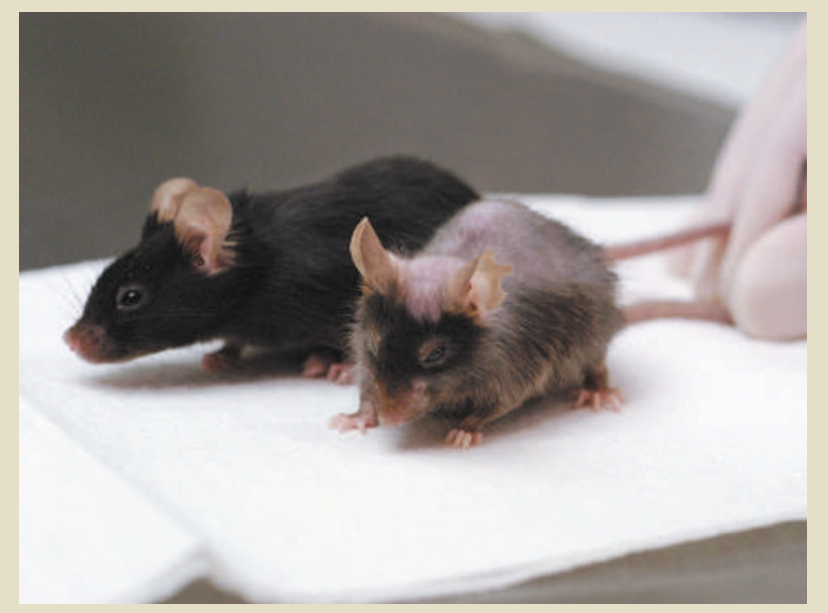

Compared with wild-type mice (left), 13-month-old POLG-defective mice (right) show a premature ageing (progeria) phenotype. Progeroid features include greying, kyphosis and reduced muscle mass.

cell death response. Active caspase-3 levels increased with ageing in wild-type mice in most tissues examined and caspase- 3 levels were significantly higher in POLG-defective mice. In situ TUNEL staining revealed that this correlated with an increase in apoptotic cells.

The concept of DNA damage contributing to ageing is not new, as mutations in several DNA repair genes cause a number of premature ageing syndromes. The findings by Kujoth et al., however, show that mtDNA mutations result in increased apoptosis in ageing tissues and that ageing can occur independently of oxidative damage. Thus, apoptosis and hence the loss of post-mitotic cells might be a dominant pathway during mammalian ageing, tipping the fine balance between cell proliferation and programmed cell death.

MYRTO RAFTOPOULOU 